\title{
Five to 15 year follow-up of resolved idiopathic central serous chorioretinopathy
}

\begin{abstract}
Purpose To recognize the long-term sequelae of resolved idiopathic central serous chorioretinopathy (ICSC) in relation to visual acuity, contrast sensitivity, and lesion size between time elapsed since disease onset. Methods Patients were recruited from fluorescein angiogram records between January 1985 and December 1995 with confirmed ICSC. Visual acuity, contrast sensitivity, and digital fundus photographs were recorded on follow-up. Comparison with the initial red-free photographs were made.

Results There was no statistically significant deterioration of visual acuity over time compared to eyes with ICSC after initial resolution and the normal eyes. The change of lesion size over time was also statistically insignificant. Contrast sensitivity compared to visual acuity showed positive correlation in both ICSC and normal eyes, but the results were statistically insignificant. Lesion size correlates negatively with visual acuity and contrast sensitivity although this was statistically insignificant.

Conclusion This study shows little, if any, correlation between time and progression of retinal pigment epitheliopathy following resolution of ICSC. Visual acuity did not seem to worsen over time. Our data were not statistically significant, but it does give insight into the natural history of what is still a relatively poorly understood disease.

Eye (2004) 18, 262-268. doi:10.1038/

sj.eye. 6700637
\end{abstract}

Keywords: central serous retinopathy; central serous chorioretinopathy; idiopathic central serous chorioretinopathy; retinal pigment epitheliopathy
R Wong, A Chopdar and M Brown

\section{Introduction}

Idiopathic central serous chorioretinopathy (ICSC) or central serous retinopathy (CSR) is a relatively poorly understood disease. There is a general understanding of epidemiology, pathology, and natural history through various studies, but most of these answers have remained theories rather than proven facts. ${ }^{1-5}$ Some remain symptom free because of subclinical attack of ICSC. When patients return years later with visual deterioration and fundal changes often to a different ophthalmologist, difficulty arises as to whether the lesion has progressed or if there is a new superimposed pathology. We compared the relation between time elapsed since disease onset and analysed lesion size, contrast sensitivity, and visual acuity hoping to provide more information to advance understanding of this mysterious disease.

\section{Materials and methods}

Patients were recruited from fluorescein angiograms recorded between January 1985 and December 1995 at East Surrey Hospital who had confirmed ICSC on fluorescein angiography. These patients were particularly chosen for their single episode of ICSC with resolution without laser treatment. A total of 106 patients were invited to attend follow-up, of which 45 patients replied and 39 attended. Age of patients ranged from 42 to 70 years old on the day of their follow-up appointment. Medical records of 23 out of 39 patients were obtained for analysis of original visual acuity. One patient was excluded from this study because he had later developed a rhegmatogenous retinal detachment in the ICSC eye.

On the day of their follow-up appointment, a brief history was taken, both eyes were examined, Snellen visual acuity and contrast sensitivity on Pelli Robson chart were measured. Dilated fundus photgraphs were also 
taken with a Topcon Digital Fundus Camera TRC 501A at $35^{\circ}$. Fellow eyes of patients who attended follow-up were allocated as 'normal' after anterior segment and fundal examination to confirm no signs of previous ICSC. The contralateral eye was confirmed to not have been affected by ICSC on presentation by analysis of fluorescein angiogram. It is difficult to assess if the contralateral eye suffered any episode of ICSC, since initial presentation as ICSC is sometimes thought to be a bilateral disease. Objective questioning aimed to prevent such occurrences.

Follow-up lesion size was measured using Topcon Imagenet 2000 software from red-free photos. The largest diameter was taken to be the maximum diameter of the area of the retinal pigment lesions seen, and lesions were chosen for their central position within the macula. Initial red-free photo taken on 35-mm film was analysed on a Tamron Fotovix Film Video Processor projected on an 11-in television screen. The measurement of original retinal pigment epithelium change was performed with a clear ruler in millimeters on the television screen.

The size of the lesions measured from 35- $\mathrm{mm}$ film was converted to 'actual lesion size' by calculating the conversion factor. This was found by dividing optic disc diameter on 35-mm film by the 'actual optic disc diameter' derived from measuring the same patient's optic disc diameter on digital photographs (Figure 1). Each patient had 'actual lesion size' calculated using individual conversion factors derived from comparing digital and 35-mm film.

Visual acuity was assessed using Snellen chart at $6 \mathrm{~m}$. Contrast sensitivity was measured using Pelli Robson chart at $1 \mathrm{~m}$ distance and constant illumination for all patients. Patients were asked to perform this test once with either eye. Unfortunately, measurement of luminance was not possible because of the lack of instruments.

Results were entered using Microsoft Excel and analysed for graphical representation and statistical analysis. All results were recorded, measured, calculated, and analysed by one investigator (RW), but precautions were taken to minimize bias.

\section{Results}

All statistical analysis was performed using the Pearson correlation test. There was no statistically significant deterioration of visual acuity over time compared to 23 eyes with $\operatorname{ICSC}\left(R=+0.11 ; R^{2}=0.014 ; P>0.05\right)$ after initial resolution and the normal eyes $(R=+0.18$; $\left.R^{2}=0.033 ; P>0.05\right)$. Although statistically insignificant, there is surprisingly minimal positive correlation with time elapsed and visual acuity in both ICSC affected and normal eyes (Figure 2a and b) (Table 1).
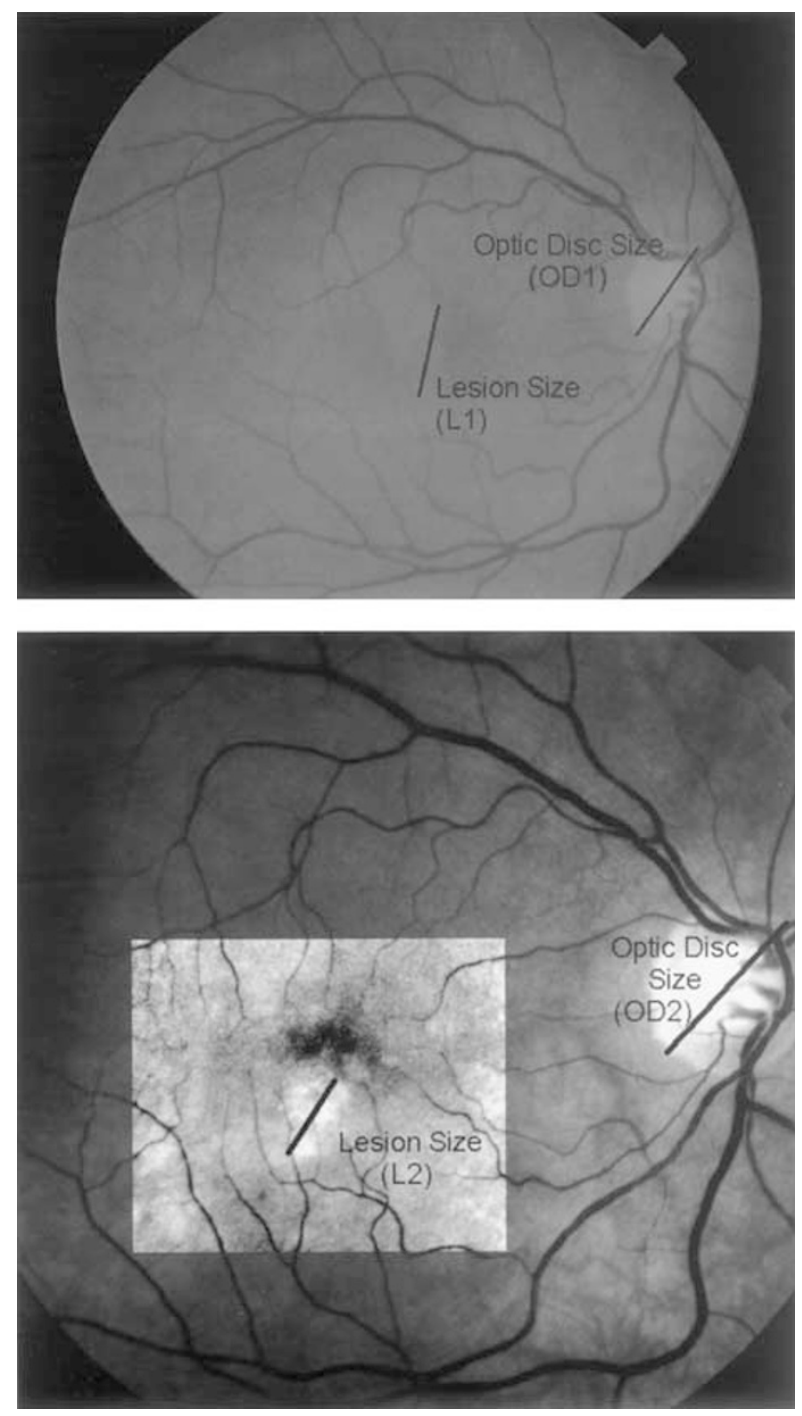

Figure 1 Original 35-mm film, follow-up digital photo (both red-free). L1: maximum diameter of lesion on 35-mm film; OD1: maximum optic disc diameter on 35-mm film; L2: maximum diameter of lesion on follow-up photo; OD2: maximum optic disc diameter on follow-up photo. Conversion: OD2/OD1 = conversion factor $(\mathrm{F})$; actual initial lesion size $=\mathrm{L} 1 / \mathrm{F}$.

The change of lesion size over time studied in 33 eyes was also statistically insignificant $(R=+0.115$; $\left.R^{2}=0.013 ; P>0.05\right)$. Again, there is minimal positive correlation over time showing possible enlargement in lesion size at follow-up, although no statistical significance can be justified (Figure 3).

Contrast sensitivity compared to visual acuity in 37 patients showed positive correlation in both ICSC $\left(R=+0.682 ; R^{2}=0.255 ; P>0.05\right)$ and normal $\left(R=+0.505 ; R^{2}=0.465 ; P>0.05\right)$ eyes. Although the evidence was statistically insignificant, there seemed to be a relatively strong correlation between the two tests of visual function. 

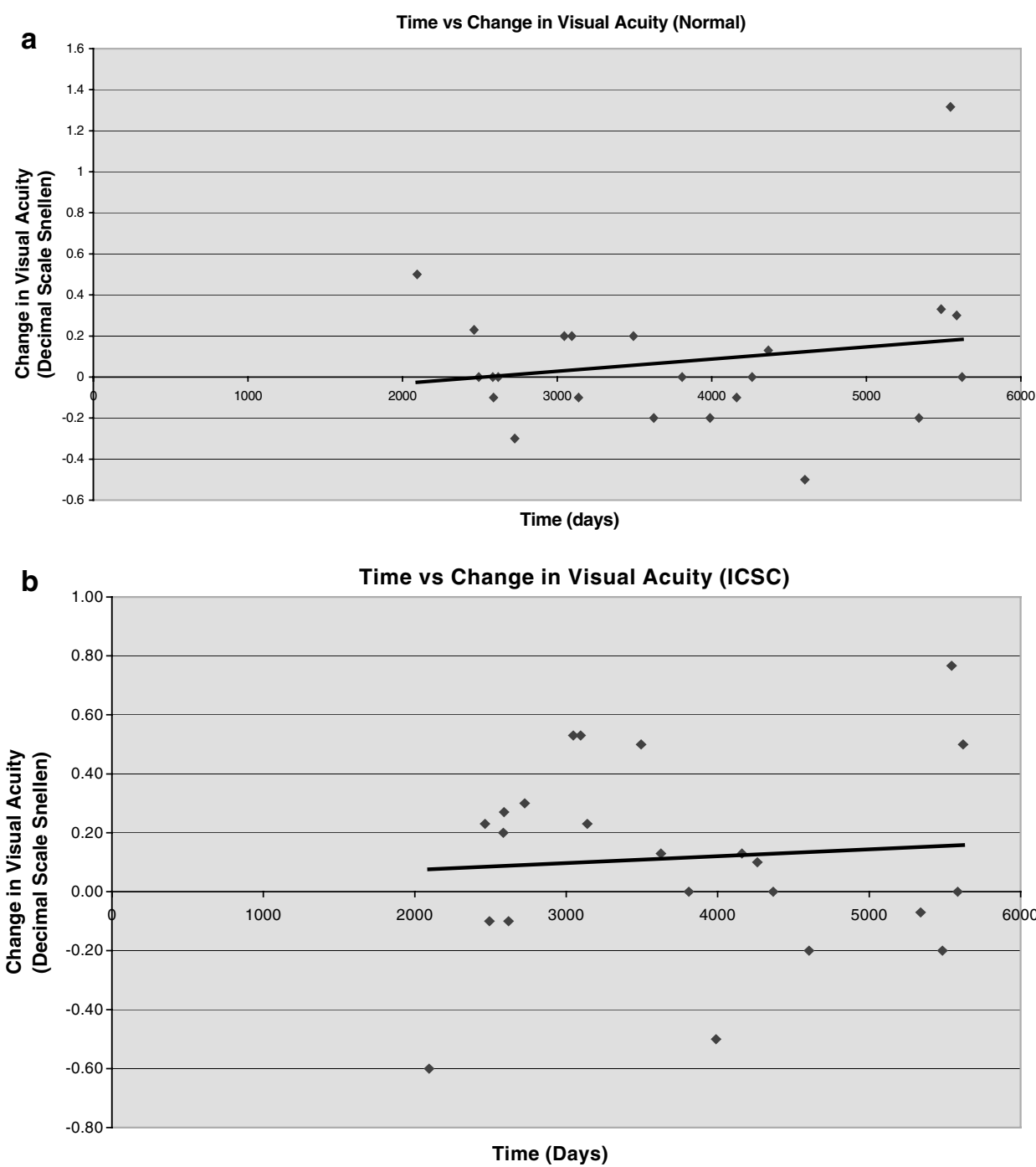

Figure 2 (a) Time $v s$ change in visual acuity (normal) and (b) time $v s$ change in visual acuity (ICSC).

Lesion size correlates negatively with visual acuity $\left(R=-0.475 ; R^{2}=0.225 ; P>0.05\right)$ and contrast sensitivity $\left(R=-0.495 ; R^{2}=0.246\right)$, although this again was statistically insignificant (Figure $4 a$ and $b$ ). It may be possible that size of retinal epithelial pigment change correlates with reduced visual acuity and contrast sensitivity.

\section{Discussion}

\section{Visual acuity vs time}

This study shows little if any correlation between time and progression of visual acuity following resolution of ICSC. Castro-Correia et $a l^{6}$ suggested that $50 \%$ of the patients may get the most severe form of the disease after 12 years of evolution. Although our data were statistically insignificant, there does not seem to be a trend showing severity with time elapsed. As the regression line shows in Figure 2a, surprisingly there is a tendency for improvement of vision over time. This may have contributed to variable background illumination. Interpretation of 'lines read' may also differ, as clinicians may well know from their experience. Furthermore, the original consultation may have been taken at different times of the disease process and therefore calculation of visual acuity change will give a false impression of improvement over time. Nevertheless, only seven of 23 $(30.4 \%)$ ICSC eyes showed deterioration of visual acuity over time and the average time of follow-up for these patients was 10.63 years compared to 10.29 years that showed no deterioration or improvement.

\section{Lesion size vs time}

Indocyanine green angiography performed by Shiraki et $a l^{7}$ in 'severe ICSC' showed persistent intrachoroidal 
Table 1 Results of visual acuity, contrast sensitivity, lesion size, and elapsed time

\begin{tabular}{|c|c|c|c|c|c|c|c|c|}
\hline$N V A 1^{a}$ & $\operatorname{ICSC} V A 1^{\mathrm{b}}$ & $N V A 2^{c}$ & $\operatorname{ICSC} V A 2^{\mathrm{d}}$ & $N C S T^{\mathrm{e}}$ & $I C S C \operatorname{CST} T^{\mathrm{f}}$ & Lesion $1^{\mathrm{g}}$ & Lesion $2^{\mathrm{h}}$ & $D A Y S^{\mathrm{i}}$ \\
\hline $6 / 6$ & $6 / 12$ & $6 / 6$ & $6 / 18$ & 1.35 & 1.2 & 1.9 & 2 & 2619 \\
\hline \multirow[t]{2}{*}{$6 / 6$} & $6 / 6$ & $6 / 4$ & $6 / 18$ & 1.65 & 1.35 & 0.5 & 2.5 & 2094 \\
\hline & & $6 / 5$ & $6 / 5$ & 1.65 & 1.65 & & & 2448 \\
\hline \multirow[t]{2}{*}{$6 / 6$} & $6 / 5$ & $6 / 5$ & $6 / 5$ & 1.65 & 1.5 & 0.2 & 0.1 & 2584 \\
\hline & & $6 / 6$ & $6 / 9$ & 1.65 & 1.35 & 1.9 & 1.8 & 4101 \\
\hline $6 / 6$ & $6 / 9$ & $6 / 5$ & $6 / 5$ & 1.5 & 1.65 & 0.7 & 1 & 3095 \\
\hline $6 / 6$ & $6 / 12$ & $6 / 6$ & $6 / 10$ & 1.65 & 1.5 & 0.7 & 0 & 5130 \\
\hline $6 / 6$ & $6 / 12$ & $6 / 5$ & $6 / 6$ & 1.5 & 1.5 & 0.5 & 2.7 & 3494 \\
\hline $6 / 9$ & $6 / 6$ & $6 / 6$ & $6 / 7.5$ & 1.2 & 1.2 & & & 5484 \\
\hline \multirow[t]{2}{*}{$6 / 6$} & $6 / 18$ & $6 / 6$ & $6 / 10$ & 1.65 & 1.2 & 4.3 & 2 & 2589 \\
\hline & & $6 / 4$ & $6 / 9$ & 1.65 & 1.35 & 1.6 & 1.58 & 2143 \\
\hline \multirow[t]{3}{*}{$6 / 9$} & $6 / 6$ & $6 / 7.5$ & $6 / 6$ & 1.65 & 1.65 & 0.1 & 1.1 & 4366 \\
\hline & & $6 / 5$ & $6 / 9$ & 1.65 & 1.65 & 0.5 & 1 & 5874 \\
\hline & & $6 / 6$ & $6 / 12$ & 1.5 & 1.05 & 0.7 & 2.4 & 4889 \\
\hline \multirow[t]{2}{*}{$6 / 9$} & $6 / 9$ & $6 / 6$ & $6 / 6$ & 1.5 & 1.35 & 0.7 & 0.6 & 3139 \\
\hline & & $6 / 10$ & $6 / 60$ & 1.05 & 1.05 & 0.5 & 3 & 4150 \\
\hline $6 / 6$ & $6 / 9$ & $6 / 6$ & $6 / 9$ & 1.35 & 1.35 & 1.1 & 0.6 & 4161 \\
\hline $6 / 6$ & $6 / 6$ & $6 / 7.5$ & $6 / 12$ & 1.5 & 1.05 & & & 3989 \\
\hline \multirow[t]{3}{*}{$6 / 5$} & $6 / 5$ & $6 / 5$ & $6 / 5$ & 1.65 & 1.65 & 0.5 & 0.3 & 3809 \\
\hline & & $6 / 6$ & $6 / 9$ & 1.35 & 1.35 & 36.1 & 4 & 4878 \\
\hline & & $6 / 6$ & $6 / 12$ & 1.65 & 1.65 & 0.4 & 0.73 & 2360 \\
\hline $6 / 5$ & $6 / 6$ & $6 / 9$ & $6 / 7.5$ & 1.65 & 1.5 & 0.5 & 2.4 & 4969 \\
\hline $6 / 4$ & $6 / 12$ & $6 / 5$ & $6 / 7.5$ & 1.65 & 1.5 & 0.5 & 2.7 & 2726 \\
\hline \multirow[t]{4}{*}{$6 / 5$} & $6 / 9$ & $6 / 6$ & $6 / 7.5$ & 1.65 & 1.5 & 0.3 & 0.9 & 3625 \\
\hline & & $6 / 5$ & $6 / 5$ & 1.65 & 1.65 & & & 4115 \\
\hline & & $6 / 9$ & $6 / 9$ & 1.5 & 1.5 & 0.7 & 0 & 3692 \\
\hline & & $6 / 6$ & $6 / 6$ & 1.5 & 1.65 & 0.7 & 1.6 & 2612 \\
\hline $6 / 6$ & $6 / 6$ & $6 / 6$ & $6 / 6$ & 1.65 & 1.5 & 1 & 0 & 2493 \\
\hline $6 / 5$ & $6 / 5$ & $6 / 4$ & $6 / 5$ & 1.65 & 1.8 & 0.4 & 0.65 & 5585 \\
\hline \multirow[t]{2}{*}{$6 / 6$} & $6 / 9$ & $6 / 7.5$ & $6 / 10$ & 1.5 & 1.5 & 1.1 & 1.85 & 5340 \\
\hline & & $6 / 18$ & $6 / 18$ & 1.2 & 1.35 & & & 5358 \\
\hline $6 / 9$ & $6 / 9$ & $6 / 6$ & $6 / 6$ & 1.5 & 1.35 & 0.9 & 1 & 2463 \\
\hline $6 / 6$ & $6 / 9$ & $6 / 5$ & $6 / 5$ & 1.65 & 1.5 & 0.8 & 0.5 & 3046 \\
\hline \multirow[t]{3}{*}{$6 / 6$} & $6 / 12$ & $6 / 6$ & $6 / 6$ & 1.65 & 1.5 & 0.3 & 0.8 & 5620 \\
\hline & & $6 / 9$ & $6 / 7.5$ & 1.65 & 1.65 & 1 & 0.55 & 5903 \\
\hline & & $6 / 6$ & $6 / 5$ & 1.5 & 1.5 & 14.7 & 1.3 & 5782 \\
\hline $6 / 9$ & $6 / 24$ & $6 / 6$ & $6 / 6$ & 1.5 & 1.65 & 0.1 & 1.2 & 5545 \\
\hline
\end{tabular}

${ }^{a} \mathrm{~N}$ VA1: normal eye visual acuity(initial consulation).

bICSC VA1: ICSC eye visual acuity (initial consulation).

${ }^{\mathrm{C}} \mathrm{N}$ VA2: normal eye visual acuity (follow-up consulation).

${ }^{d}$ ICSC VA2: ICSC eye visual acuity (follow-up consulation).

${ }^{e} \mathrm{~N}$ CST: normal eye contrast sensitivity (follow-up consulation).

fICSC CST: ICSC eye contrast sensitivity (follow-up consulation).

'Lesion 1: lesion size (initial consulation).

${ }^{\mathrm{h}}$ Lesion 2: lesion size (follow-up consulation).

${ }^{i}$ Days: days elapsed between initial and follow-up consulation.

leakage of dye in all four patients reviewed over 2-3 years. In view of this evidence, we believe that there would be enlargement of retinal pigment epitheliopathy over time. As Figure 3 shows there is positive correlation between enlargement of lesion size and time elapsed although, as previously noted, the correlation was statistically insignificant. From our sample of 28 eyes, $17(60.7 \%)$ showed average lesion size enlargement of $1.1 \mathrm{~mm}$, while 11 of 28 eyes (39.3\%) showed no change or shrinkage of lesion size. Although enlargement in size can be explained by persistent leakage and chronic progression, shrinkage is more difficult to theorize in terms of pathological mechanism. These results may be due to aberrations in calculation of conversion from 35$\mathrm{mm}$ film to actual lesion size and comparing lesion size measured from digital angiography. Furthermore, position of lesion was not taken into consideration in this study, which may contribute to the results of our measurement. As noted by studies from Shiraki $e^{2} a l^{7}$ and Levine $e t a l^{8}$ they have shown new retinal pigment 


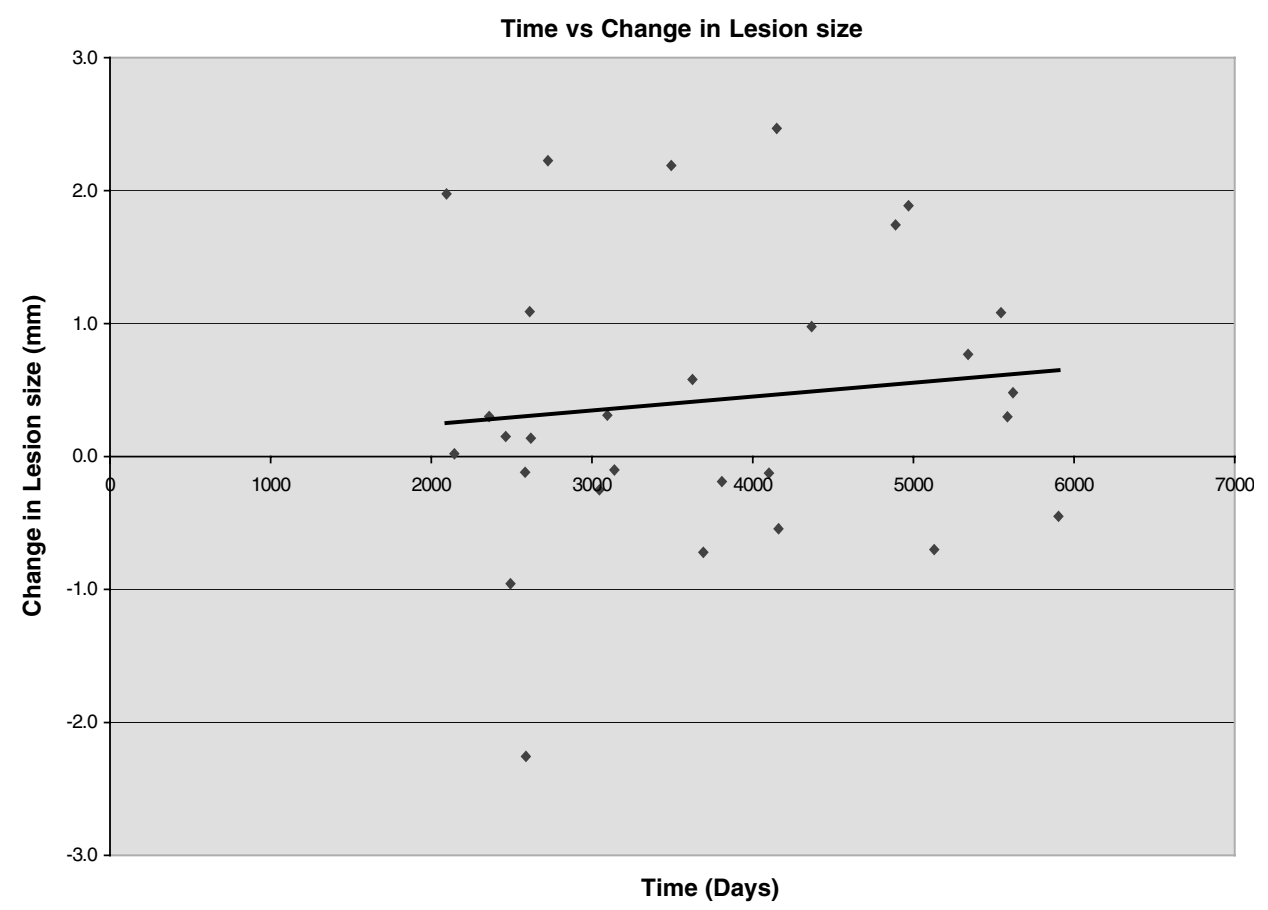

Figure 3 Time $v s$ lesion size.

epithelial defects outside the areas of previous serous detachment and shift in location of intrachoroidal leakage.

\section{Contrast sensitivity vs visual acuity}

On the follow-up consultation, we also measured contrast sensitivity using the Pelli-Robson chart. Previous studies of contrast sensitivity following resolution of ICSC by Koskela et $a l^{9}$ showed statistically significant correlation between visual acuity and contrast sensitivity using the Vistech test and Wavetek 143 function generator. Mutlak et al ${ }^{10}$ also found lower sensitivity following resolved ICSC. We also found a strong correlation, although statistically insignificant, of visual acuity and contrast sensitivity in both normal and ICSC affected eyes using the Pelli-Robson chart.

Maaranen and Mantyjarvi ${ }^{11}$ used Pelli-Robson chart to study contrast sensitivity reduction in eyes, which showed normal resolution of visual acuity following ICSC. We found that of the nine patients who regained visual acuity of $6 / 6$ or better, five patients had a reduction in contrast sensitivity while three had unequivocal measurements compared to the fellow eye, and one had worse contrast sensitivity in the fellow eye.

\section{Lesion size vs visual acuity + contrast sensitivity test}

We also analysed the size of lesion and its effect on visual acuity. Koskela et $a l^{9}$ noted that ultimate clinical picture of the macula did not correlate with contrast sensitivity. Although our correlation was not of statistical significance, there was a negative correlation shown as seen in Figure $4 \mathrm{~b}$. There was a similar correlation when comparing lesion size and visual acuity (Figure 4a). Perhaps this is a genuine correlation, but it is difficult to assess its significance as the design of this study was such that the patient was examined and measured for visual acuity and contrast sensitivity, followed by digital fundus photography and data analysis, all by the same examiner. However, the intentions of producing an accurate and unbiased result were genuine.

The study may seem biased in view of the methods of data collection and analysis, but all measures were made to minimize these errors relative to the resources available. Measurements of initial 35- $\mathrm{mm}$ film was carried out on separate days and recorded on a separate field without knowledge of performance on visual acuity or contrast sensitivity. Further measurements from follow-up digital photography were performed again on separate field without knowledge of initial lesion size or visual performance. Red-free photos were used on both occasions to analyse lesion size as fluorescein angiograms can overestimate retinal epithelial changes. No attempt was made to repeat fluorescein angiography on asymptomatic patients because of the unethical nature of the procedure.

It was also impossible to distinguish if the same cohort had any signs of age-related macular degeneration. Our sample patients had reached their 60s with one patient 

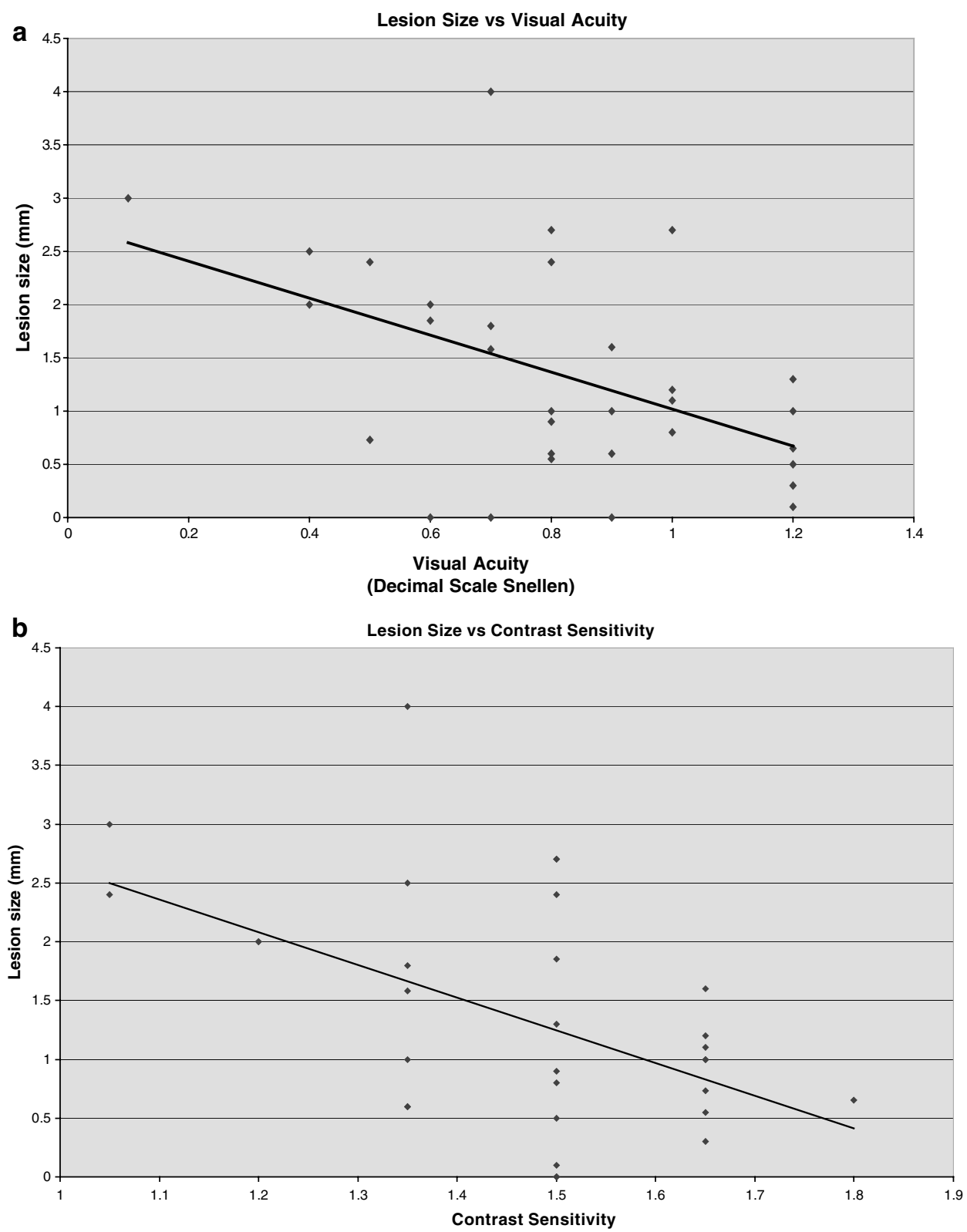

Figure 4 (a) Lesion size $v s$ visual acuity and (b) lesion size vs contrast sensitivity.

who was 72 years old by the time of re-evaluation. Since the retinal pigment epithelial changes of ICSC mimic those of age-related macular degeneration in appearance and location, we have only assumed there were little, if any, effects of age-related macular degeneration in the affected eye by examining the fellow eye.

The statistical insignificance of the results was disappointing, but there were general comments that could be made regarding the various relations found in our study. Although most ICSC patients do not undergo fluorescein angiography, all 106 patients recruited were confirmed to have ICSC on fluorescein angiograms. Only
39 of 106 patients invited attended due to the fluidity of the local population and the relatively young age at which typical ICSC presents. Of the 106 invited, 45 replied, three did not want further follow-up, two did not attend for their follow-up, one died, and 54 either did not reply or had moved. A larger sample size may have aided our efforts and possibly a multicenter study may be recommended. We suggest further follow-up in a further 5 or 10 years time of the same cohort to provide further data on the outcome of ICSC. While little is known about the pathogenesis and aetiology of ICSC, most cases resolve spontaneously. As ophthalmologists, 
it is common for us to trivialize the situation as patients sometimes suffer the consequences following presumed resolution of the disease. During the recruitment of patients, one patient alerted me to an Internet chat forum, where fellow patients discussed their experience and theories regarding the aetiology of ICSC. There were many who continued to suffer the consequences of 'resolved ICSC' and were disappointed about the manner in which they were advised by their ophthalmologist regarding the final visual outcome. Hopefully, this paper promotes awareness in the counseling of patients following an episode of ICSC.

\section{References}

1 Ulbig MR, Riordan-Eva P, Holz FG, Rees HC, Hamilton PA. Membranoproliferative glomerulonephritis type II associated with central serous retinopathy. Am J Ophthalmol 1993; 116: 410-413.

2 Oosterhuis JA. Familial central serous retinopathy. Graefes Arch Clin Exp Ophthalmol 1996; 234: 337-341.

3 Zamir E. Central serous retinopathy associated with adrenocorticotrophic hormone therapy. A case report and a hypothesis. Graefes Arch Clin Exp Ophthalmol 1997; 235: 339-344.
4 Chuang EL, Sharp DM, Fitzke FW, Kemp CM, Holden AL, Bird AC. Retinal dysfunction in central serous retinopathy. Eye 1987; 1: 120-125.

5 Faurschou S, Rosenberg T, Nielsen N. Central serious retinopathy and presenile disciform exudative macular degeneration. Is there an aetiological relationship between these two exudative conditions of the macula? Acta Ophthalmol 1977; 55: 515-524.

6 Castro-Correia J, Coutinho MF, Rosas V, Maia J. Long-term follow-up of central serous retinopathy in 150 patients. Doc Ophthalmol 1992; 81: 379-386.

7 Shiraki K, Mariwaki M, Matsumoto M, Yanagihara N, Yasunari T, Miki T. Long-term follow-up of severe central serous chorioretinopathy using idocyanine green angiography. Int Ophthalmol 1997-1998; 21: 245-253.

8 Levine R, Brucker AJ, Robinson F. Long-term follow-up of idiopathic central serious chorioretinopathy by fluorescein angiography. Ophthalmology 1989; 96: 854-859.

9 Koskela P, Laatikainen L, von Dickhoff K. Contrast sensitivity after resolution of central serous retinopathy. Graefes Arch Clin Exp Ophthalmol 1994; 232: 473-476.

10 Mutlak JA, Dutton GN, Zeini M, Allan D, Wail A. Central visual function in patients with resolved central serous retinopathy. A long term follow-up study. Acta Ophthalmol 1989; 67: 532-536.

11 Maaranen T, Mantyjarvi M. Contrast sensitivity in patients recovered from central serous retinopathy. Int Ophthalmol 1999; 23: 31-35. 